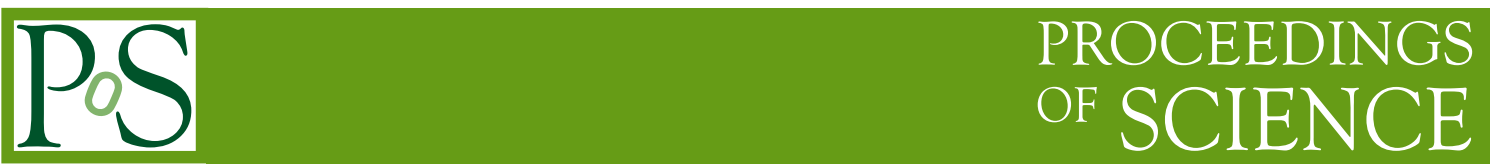

\title{
Searching for extended Pair Halo emission
}

\section{Lisa Fallon}

Dublin Institute for Advanced Studies

E-mail: Ifallon@cp.dias.ie

\section{- on behalf of the H.E.S.S. Collaboration}

A search has been conducted for the giant Pair Halo structures which are inevitably formed around $\mathrm{TeV}$ sources due to interactions of very high energy gamma-rays with the Extragalactic Background Light (EBL). The resulting electron/positron pairs in turn Compton upscatter photons of the 2.7 K Cosmic Microwave Background Radiation to produce a second generation of gamma-rays which again interact with the EBL; thus an electromagnetic cascade develops. If the magnetic fields on Mpc scales surrounding the central source are sufficiently strong $\left(10^{-11} \mathrm{G}\right.$ or more), electrons are effectively isotropised before interacting with radiation fields. In this case, an extended halo is produced around the source. Using H.E.S.S. observations of Active Galactic Nuclei, including data from PKS 2155-304, 1ES 1101-232 and 1ES 0229+200, we are currently undertaking a detailed analysis of these sources. The preliminary results are presented here.

25th Texas Symposium on Relativistic Astrophysics

December 6-10, 2010

Heidelberg, Germany 


\section{Introduction}

High energy gamma rays have a limited mean free path in the universe due to interactions with the intergalactic soft photon radiation, resulting in pair production [1]. Giant electron-positron structures which are formed due to the development of pair cascades, initiated by interactions of primary multi-TeV photons from high energy extragalactic sources with the extragalactic background photon fields, are known as Pair Halos. The physical model for Pair Halos was originally presented by Aharonian, Coppi and Völk [2]. Depending on the magnetic fields in the vicinity of the source, the cascade can result in an isotropic halo around an initially beamed source.

The two principal processes which initiate the formation of Pair Halos around extragalactic objects are photon-photon pair production (PP) and inverse Compton Scattering (IC).

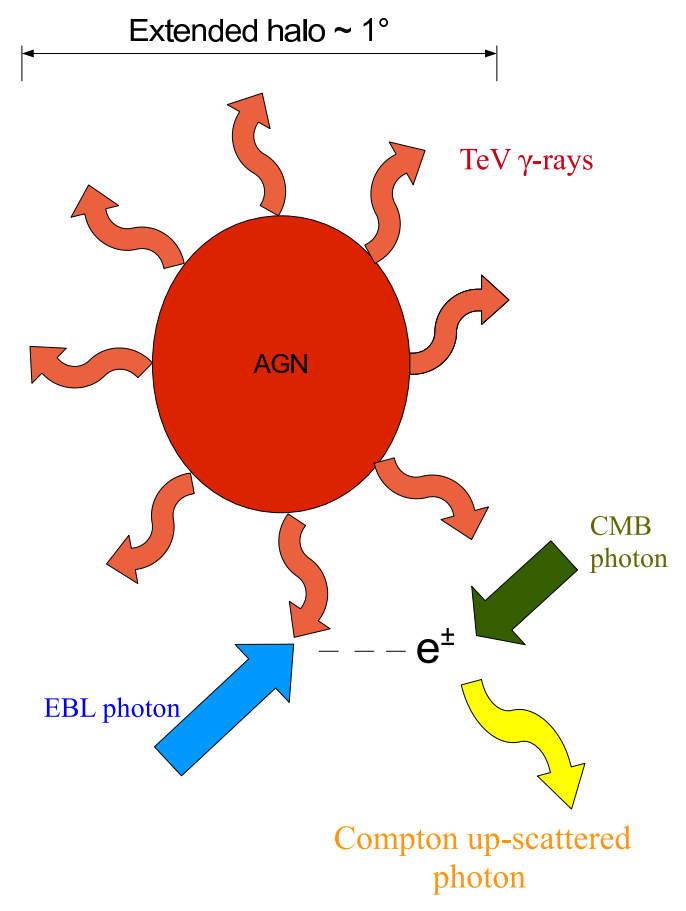

Figure 1: A simple cartoon of the Pair Halo physical model (not to scale). The red arrows represent very high energy gamma rays leaving the AGN, which interact with the Extragalactic Background Light (EBL), shown here are as blue arrow. They pair produce and these electrons and positrons $\left(e^{ \pm}\right)$compton upscatter with the Cosmic Microwave Background (CMB) (green arrow) producing a upscattered photon (yellow arrow).

When very energetic $\gamma$ rays interact with background photons (e.g. Cosmic Microwave Background photons), their energy is not lost. The two interacting photons convert into a electronpositron pair $\left(\mathrm{e}^{+} \mathrm{e}^{-}\right.$) (see Fig. 1). These new pairs will create new $\gamma$-rays by inverse Compton scattering on the background field photons. Again, these new $\gamma$ rays can pair produce and this process continues, developing an electromagnetic cascade. The cascade ends when the comptonupscattered photons no longer have enough energy to pair-produce on the background photons. 
Essentially, from a single very high energy gamma ray, through the cascading process, the energy of the initial photon is transformed into many photons of a much lower energy.

The formation of a Pair Halo depends on two criteria. First of all, the source must have an extended gamma-ray spectrum up to $\mathrm{TeV}$ energies. The initial gamma rays must have a sufficiently high energy to interact with the Extragalactic Background Light (EBL). Secondly, there must be a relatively high magnetic field $\left(\mathrm{B} \geq 10^{-11} \mathrm{G}\right.$ ) within $\sim 10 \mathrm{Mpc}$ of the source, to facilitate isotropic emission and create the "halo effect". If these two requirements are met, cascade radiation emitted isotropically from the source should be observable at energies below a few $\mathrm{TeV}$.

The preferred distance for observation of a Pair Halo with the H.E.S.S. experiment, due to its field of view, is in the range of several megaparsecs $(\mathrm{Mpc})$ to around 1 gigaparsec $(\mathrm{Gpc})$. This would allow for a reduction in the emission angle and still maintain a high enough flux for detection. If the source is too close, it becomes almost impossible to distinguish between the halo photons and background radiation.

Although some attempts have been made to observe this phenomenon (by HEGRA in 2001 [3] and MAGIC in 2009 [ [ఫ]) none have been successful to date. Here H.E.S.S. observations of the Active Galactic Nuclei : PKS 2155-304, 1ES 1101-232 and 1ES 0229+200 are discussed, in an attempt to observe or constrain Pair Halo emission.

\section{H.E.S.S. Observations and Analysis}

The H.E.S.S. experiment has been in operation since June 2002 and is located in the Khomas Highlands of Namibia ( $23^{\circ} 16^{\prime} 18^{\prime}$ ' S, 16 30' 1” E), $1835 \mathrm{~m}$ above sea level [5]. It is an array of four Imaging Atmospheric Cherenkov Telescopes (IACT) which detects cosmic gamma rays in the $100 \mathrm{GeV}$ to $100 \mathrm{TeV}$ energy range. The four H.E.S.S. telescopes are placed in a square formation with a side length of 120 metres. The effective mirror surface area per telescope is $107 \mathrm{~m}^{2}$. The total field of view of the detector is $5^{\circ}$ in diameter. For this analysis, the standard H.E.S.S. data quality selection criteria [6] were applied to each source. We imposed the further criterion that only runs which had data from all 4 telescopes would be used, to improve the overall angular resolution. The Reflected-Region method [㣙] was used for the estimation of the background.

\subsection{PKS 2155-304}

The AGN PKS 2155-304 which is located at a redshift of $z=0.117$, has been detected by the H.E.S.S. array with high significance at energies greater than $160 \mathrm{GeV}$ [8]. This source has been well studied and has a long history of strong broad-band emission. At present there are over 200 hours of data collected by the H.E.S.S. experiment for this source. With such a vast collection of data to hand, it allows us to divide the data set and focus on different aspects. In 2006, there was an exceptional flare [9], which we have chosen to look at separately from the rest of the data, creating two data sets: high state and low state. Removing the flare from the main data set allows us to focus on this source in a low state, where a halo should be more easily detectable. The data set for the low state amounts to $\sim 174$ hours, only including data of good quality. Focusing solely on the exceptional flare from 2006, we have a data set of 34 runs ( $\sim 16$ hours). These observations were taken during the nights of July 29th to 31st 2006 (3 nights in total). 


\subsection{ES 1101-232}

This blazar was first observed with the H.E.S.S. array in 2004 at VHE energies $\left(\mathrm{E}>10^{-11}\right.$ eV) [10]. Its redshift was determined to $z=0.186$ [11]. We have analysed 67.2 hours (144 runs of $\sim 28 \mathrm{~min}$ each) of good quality data for this source at a significance level of $11 \sigma$. Our data set spans observations from 2004 to 2008.

\subsection{ES 0229+200}

This source was first observed by H.E.S.S. in late 2004 and was initially detected with a signal of $6.6 \sigma$ [12]. It is located at a redshift of $z=0.1396$ [13]. We have accumulated a total of 79.3 hours (170 runs) for this source between 2004 and 2009. Similarly to 1ES 1101-232, we chose runs which had data from all four telescopes.

\subsection{Theoretical Model}

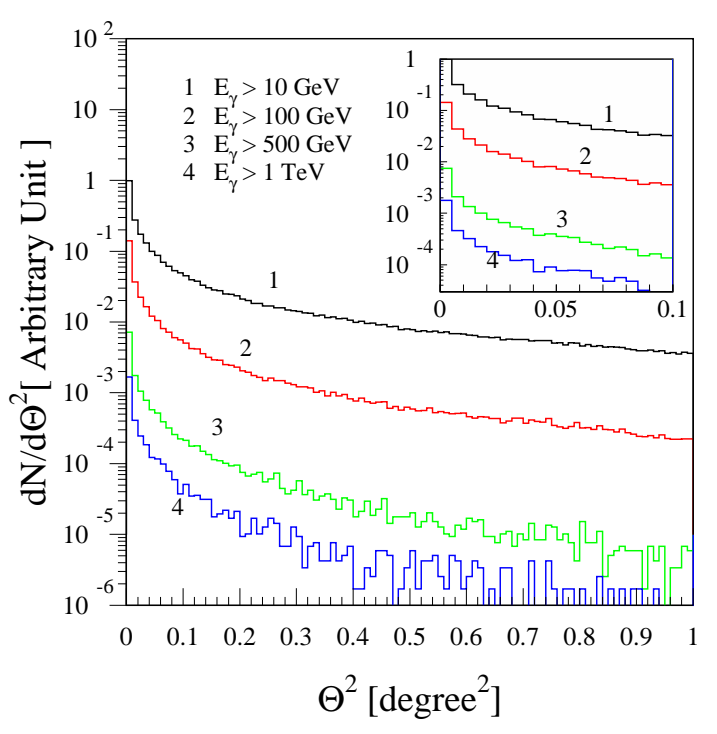

Figure 2: Differential angular distributions of pair halo emission at $\mathrm{z}=0.129$ (in arbitrary units) taken from [14].

A detailed study of Pair Halo emission has been undertaken by Eungwanichayapant \& Aharonian (2009) and Fig. 目 is taken from this publication [14]. It illustrates the theoretical predictions for pair halo angular distributions for different energy cuts. We will profit from this comprehensive study and use it as part of our future analysis as a comparison to H.E.S.S. observations with the most up-to-date theory.

\section{Preliminary Results}

PKS 2155-304 exhibited an exceptional flare in 2006 which had an average VHE flux observed by H.E.S.S. more than 10 times its typical value and was varying on minute timescales. Causality requires that PKS 2155-304 is a point-like source for H.E.S.S. during the flare state, which makes 


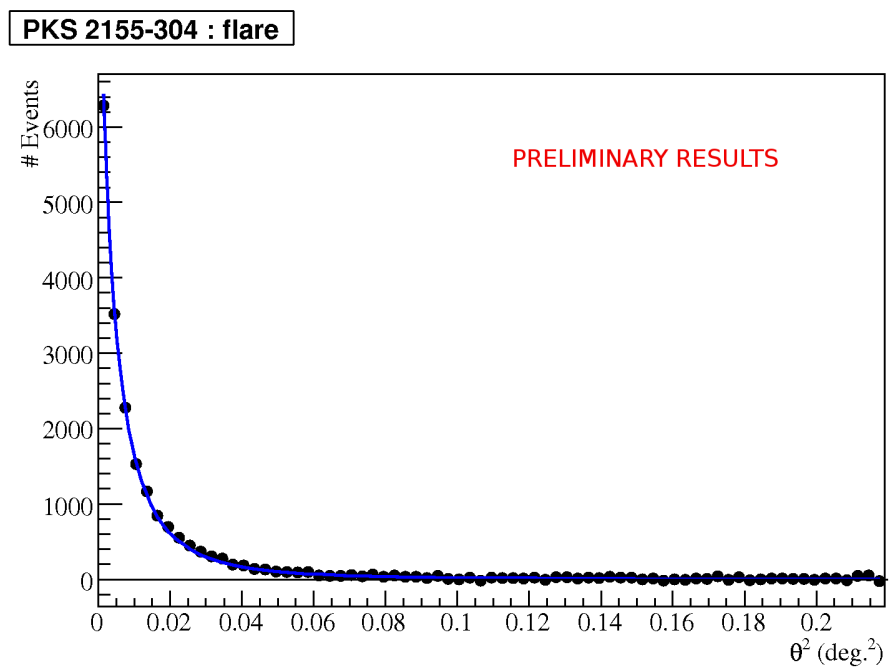

Figure 3: PKS 2155-304 flare data set fitted with the H.E.S.S. Point Spread Function from Monte Carlo simulations in blue.

it an ideal candidate to conduct systematic studies on the H.E.S.S. point spread function. Another advantage of using the flare to study the systematics, is the exceptionally good signal/noise ratio we obtained for this data set. Using Monte Carlo simulations for different offset and zenith angles,

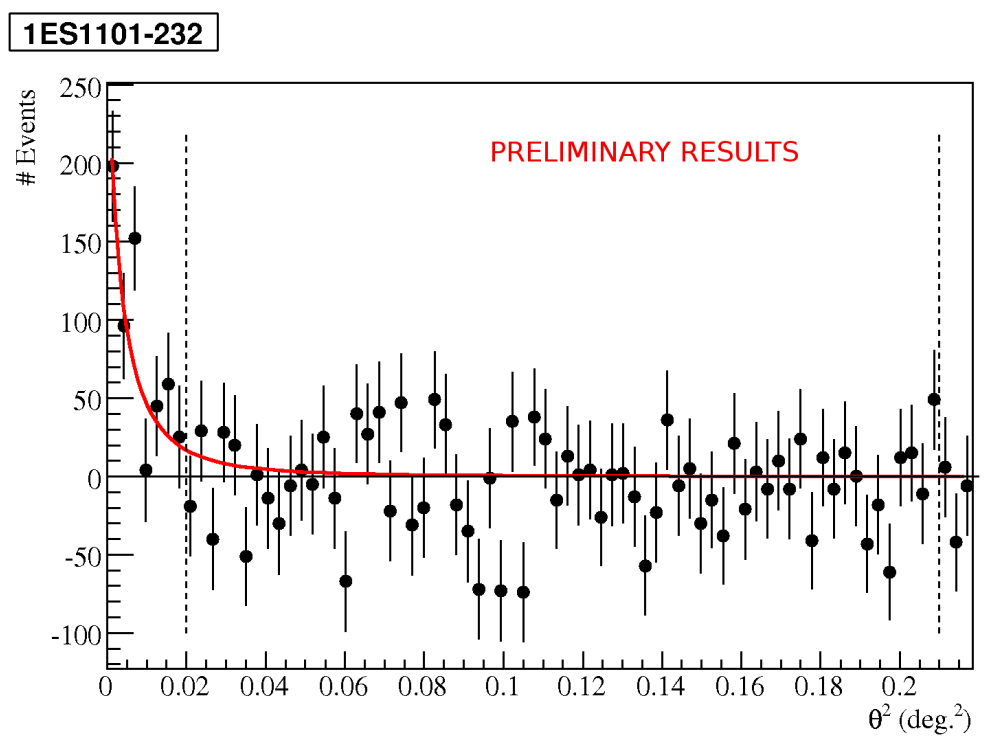

Figure 4: 1ES 1101-232 with the H.E.S.S. Point Spread Function in red. The vertical dashed line at 0.02 denotes the standard selection cut for point sources used by H.E.S.S..

which are weighted to match the data set and the source spectrum, we fit them with a new function (a simple double gaussian) and then finally fit this to the data with only the normalisation left free. This is done with the intention of verifying our understanding of the PSF. As can be seen in Fig. 3, it is a very good fit to the $\theta^{2}$ distribution of the excess events from the PKS 2155-304 flare data set. 
The full details will be explicitly discussed in a forthcoming publication.

For each of our sources (PKS 2155-304 low state, 1ES 1101-232 and 1ES 0229+200), we focused on the angular distribution of the emission, knowing that a deviation from the standard point source profile would be a strong hint of a halo excess. As can be seen in Fig. औ, there is no compelling evidence of pair halo excess. This is true for each of these three sources. The necessary analysis to obtain the most constraining upper limits possible on an extended pair halo flux component for each source is currently being conducted. Two different approaches to the calculating the upper limits have been used. We have used a model dependent approach assuming a halo profile taken from the results of Eungwanichayapant and Aharonian [14]. We have also performed a less-sensitive, model-independent approach which focuses on the region between $\theta_{[1]}^{2}$ and $\theta_{[2]}^{2}$, i.e between 0.02 and 0.22 in Fig. $甘$, comparing the over-all excess to a point source profile.

A more detailed analysis and a comprehensive discussion will be presented in an upcoming publication which is currently under preparation.

\section{References}

[1] Gould, R.J., \& Schreder, G, Opacity of the Universe to High-Energy Photons, Physics Review Letters 16252 (1966)

[2] Aharonian, Coppi \& Völk Very high energy gamma rays from active galactic nuclei: Cascading on the cosmic background radiation fields and the formation of Pair Halos, Astrophysical Journal 423 L5-L8 (1994)

[3] Aharonian, et al., Search for a TeV gamma-ray halo of Mkn 501, Astronomy \& Astrophysics 366746 (2001)

[4] J. Aleksić et al., Search for an extended VHE $\gamma$-ray emission from Mrk 421 and Mrk 501 with the MAGIC Telescope, Astronomy and Astrophysics 524 A77 (2010)

[5] J.A. Hinton, The status of the H.E.S.S. project, New Astronomy Review 48331 (2004)

[6] F. Aharonian et al., Observations of the Crab nebula with H.E.S.S., A\&A 457899 (2006)

[7] D. Berge et al., Background modelling in very-high-energy $\gamma$-ray astronomy, A\&A 4661219 (2007)

[8] Aharonian et al.,H.E.S.S. observations of PKS 2155-304, A\&A 430865 (2005)

[9] Aharonian et al., Simultaneous multiwavelength observations of the second exceptional $\gamma$-ray flare of PKS 2155-304 in July 2006, A\&A 502 749A (2009)

[10] Aharonian et al., Detection of VHE gamma-ray emission from the distant blazar 1ES 1101-232 with H.E.S.S. and broadband characterisation, A\&A 470 475A (2007)

[11] R. A. Remillard, et al, Two X-ray-selected BL Lacertae objects observed with the HEAO 1 scanning modulation collimator, ApJ 345140 (1989)

[12] Aharonian et al., New constraints on the mid-IR EBL from the H.E.S.S. discovery of VHE $\gamma$-rays from IES 0229+200, A\&A 475L 9A (2007)

[13] J. H. Woo et al., Black Hole Masses and Host Galaxy Evolution of Radio-Loud Active Galactic Nuclei, ApJ 631762 (2005)

[14] A. Eungwanichayapant and F. Aharonian, Very High Energy $\gamma$-rays from $e^{ \pm}$Pair Halos, International Journal of Modern Physics D 18911 (2009) 